\title{
O MÉTODO DE CAVALIERI E A ÁREA SOB A CICLÓIDE: APONTAMETOS
}

\author{
THE CAVALIERI METHOD AND THE AREA UNDER THE CYCLOID: POINTS
}

\section{ROGERIO LUIZ QUINTINO DE OLIVEIRA JUNIOR ${ }^{a}$}

\begin{abstract}
Resumo
Este artigo tem por objetivos principais apresentar um breve relato histórico de um resultado muito usado em geometria: o Princípio de Cavalieri, além de uma das mais belas demonstrações já feitas, à época de sua descoberta: o valor da área sob a ciclóide, por Roberval. É notório como este princípio é mostrado aos alunos sem nenhuma argumentação plausível, na maioria das vezes. Isto ocorre muito por falta de preparo do próprio professor de Matemática. Desta forma, este trabalho pretende também relembrar a definição da ciclóide e despertar o interesse do professorleitor neste tão importante método da Matemática para que ele se sinta capaz de promover uma aprendizagem significativa em seus alunos e despertar seu próprio interesse em produzir aulas com conteúdos que fujam do ensino tradicional já tão defasado.
\end{abstract}

Palavras-chave: Ensino, História da Matemática, Princípio de Cavalieri.

\begin{abstract}
The main objectives of this article are to present a brief historical account of a result widely used in geometry: the Cavalieri's Principle, in addition to one of the most beautiful demonstrations ever made at the time of its discovery: the value of the area under the cycloid, by Roberval. It is notorious how this principle is shown to students without any plausible argument, most of the time. This is largely due to the lack of preparation of the mathematics teacher himself. In this way, this work also intends to remember the definition of the cycloid and awaken the interest of the teacher-reader in this very important method of Mathematics so that he feels capable of promoting meaningful learning in his students and awakening his own interest in producing classes with content that flee from traditional education already so outdated.
\end{abstract}

Keywords: Teaching, History of Mathematics, Cavalieri's Principle.

MSC2010: 97D10

\footnotetext{
a Universidade Estadual do Rio de Janeiro, Rio de Janeiro, Brasil; ORCID: 0000-0002-9894-9980. E-mail: roger.quintino@hotmail.com
} 


\section{Introdução}

Já não é de hoje que a História da Matemática tem entrado de maneira bastante interessante como um instrumento facilitador no ensino-aprendizagem de Matemática. Conhecer um pouco de como os antigos matemáticos pensavam, como era a simbologia de sua época, entre outras coisas, podem fomentar o interesse dos alunos em aprender e discutir o conteúdo que estão aprendendo em sala.

Uma primeira forma diz respeito às possibilidades de se recorrer à história como um recurso pedagógico adicional, isto é, como meio auxiliar, potencialmente rico, para se promover e repensar o ensino-aprendizagem de matemática. (MIGUEL, 1993, p. 12)

A aula tradicional de matemática com lousa e giz, conteúdos prontos e regras para serem memorizadas não só desmotivam os estudantes como causam um enorme despreparo para aqueles que pretendem cursar alguma área de ciência exatas.

Em relação à interdisciplinaridade entre História e Matemática, Lemos (2012) diz que

\footnotetext{
Nos nossos dias, o ensino da matemática tende frequentemente para a aplicação "mecanicista" de técnicas apoiada numa notação eficiente, orientada para a resolução de exemplos e problemas, sem estimular uma compreensão suficientemente profunda das ideias e dos conceitos. $\mathrm{O}$ estudo da história da matemática é uma excelente forma de mitigar esta deficiência. Permite-nos compreender a génese e o desenvolvimento das grandes ideias, tal como surgiram e tomaram forma na mente dos grandes matemáticos. (LEMOS, 2012, p. 66)
}

Observando tudo isso, este trabalho propõe dar luz à um princípio bastante conhecido da Geometria: o do italiano Bonaventura Cavalieri.

Este princípio é ensinado na Educação Básica, mas pretendemos fazer um pequeno resumo de como ele apareceu na esteira da descoberta do Cálculo Diferencial e Integral, no século XVII. Além disso, como um segundo objetivo principal, mostraremos um dos mais belos problemas resolvidos por este método à sua época: o da descoberta da medida da área sob a ciclóide, por Roberval.

Com os objetivos acima delineados, procederemos à uma breve discussão histórica do Método de Cavalieri seguida de uma revisão da construção da ciclóide. Finalmente, veremos com detalhes a prova de Roberval descrita acima e faremos nossas considerações finais. 


\section{O Método dos Indivisíveis de Cavalieri}

Nos primórdios da descoberta do Cálculo Diferencial e Integral por Newton e Leibniz, de maneira distinta, os matemáticos do início do século XVII certamente estavam bastante empolgados com os vários métodos existentes para encontrar tangentes e normais a certas curvas, áreas e volumes de figuras etc. Nesta época, apesar de vários matemáticos usarem alguns métodos diferentes ao mesmo tempo, podemos distinguir três (GRAY, 1987, p. 12):

(a) Métodos contendo a ideia de elementos indivisíveis;

(b) Métodos que recaíam em elementos infinitesimais de área ou volume;

(c) Métodos recaindo em elementos finitos de volume e algum tipo de argumento de aproximação.

Estamos interessados, neste artigo, no primeiro desses métodos, em especial no Método de Cavalieri. Apesar de ele ser um método que, por exemplo, aproxima áreas por uma infinidade de linhas, ele causa certo desapontamento pois linhas não possuem área e existem exemplos que mostram que este método é falho: veja, por exemplo Gray (1987, p. 12). Outro fato interessante a se destacar é que as ideias por detrás dos métodos dos indivisíveis eram geralmente pouco conhecidas.

Esta quase situação paradoxal é principalmente causada pelo fato dos autores lidarem em geral com o desenvolvimento da análise no século XVII tomando Cavalieri como um ponto natural de começo, mas não discutindo seu método em detalhes, porque seu principal objetivo era traçar ideias sobre infinitesimais. Houve uma tendência de apresentar os fundamentos de seu método de uma forma que é bem simplificada para refletir as intenções originais de Cavalieri. (ANDERSEN, 1985, p. 292, tradução nossa) ${ }^{\mathrm{b}}$

No entanto, Cavalieri propôs um Princípio baseado nos indivisíveis que funciona até hoje. Ele diz que:

\footnotetext{
b No original: The ideas underlying this method, however, are generally little known.' This almost paradoxical situation is mainly caused by the fact that authors dealing with the general development of analysis in the 17th century take CAVALIERI as a natural starting point, but do not discuss his rather special method in detail, because their aim is to trace ideas about infinitesimals. There has even been a tendency to present the foundation of his method in a way which is too simplified to reflect CAVALIERI'S original intentions.
} 
"O princípio assegura que duas figuras planas possuem a mesma área se elas estão entre mesmas paralelas, e cada linha desenhada paralelamente às duas linhas dadas corta em cordas iguais cada figura." (GRAY, 1987, p. 13, tradução nossa) ${ }^{c}$

Abaixo, temos uma figura mostrando o Princípio de Cavalieri. Observe que as figuras estão contidas entre os dois mesmos eixos e cada seção plana contida na primeira figura tem a mesma dimensão que na outra figura sob o mesmo segmento plano. Logo, pelo Princípio as figuras devem ter a mesma área.

Figura 1 - O Princípio dos Indivisíveis de Cavalieri

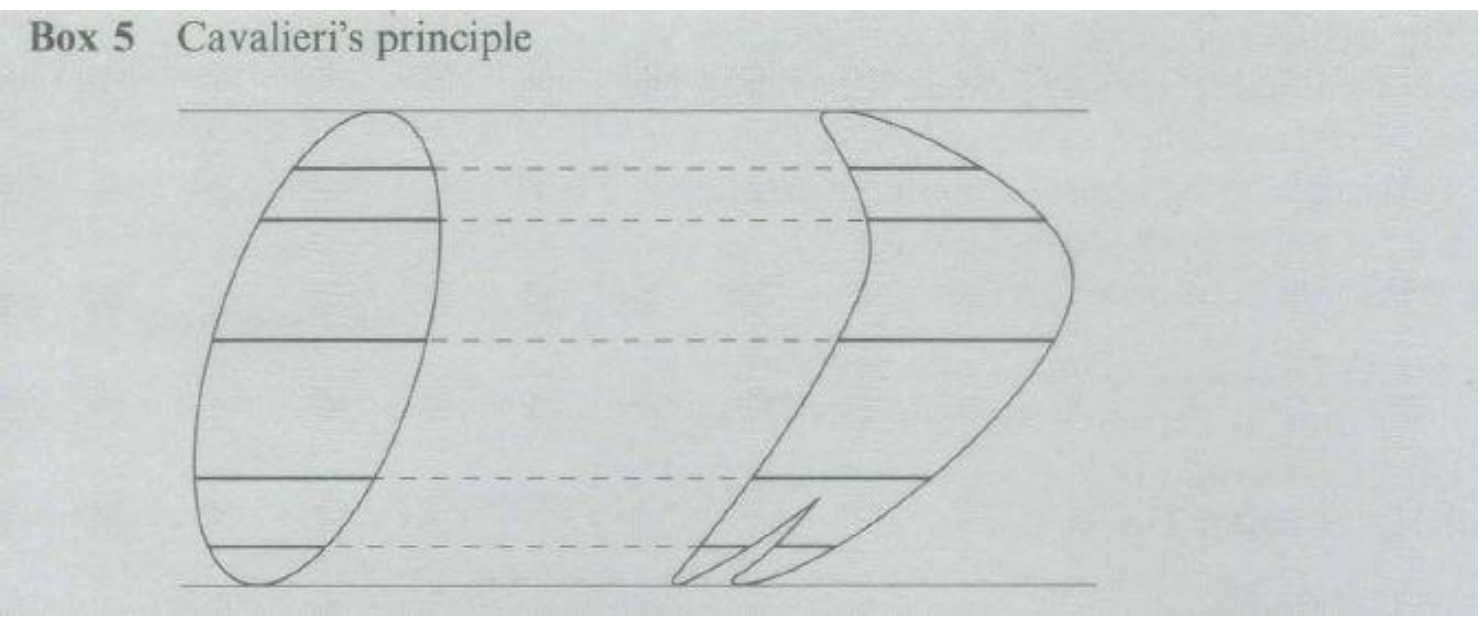

Fonte: GRAY, p. 13.

Uma observação que podemos fazer sobre este método é que "É impossível, no entanto, provar este Teorema com tanto rigor quanto ele é visto em qualquer demonstração envolvendo a teoria de limites; [...]." (EVANS, 2017, p. 447, tradução nossa $)^{\mathrm{d}}$.

Já dissemos, anteriormente, que este método é usado até hoje, mesmo em três dimensões. Por exemplo, no Ensino Básico aprendemos a calcular o volume de uma esfera usando este princípio.

Neste caso, os sólidos devem estar contidos entre os mesmos dois planos paralelos e cada seção plana de uma figura em um sólido deve ter a mesma área que a segunda no outro sólido. Assim, pelo Princípio de Cavalieri, os sólidos devem ter volumes iguais.

\footnotetext{
${ }^{\mathrm{c}}$ No original: The principle asserts that two plane figures have the same area if they are between the same parallels, and any line drawn parallel to the two given lines cuts off equal chords in each figure.

d No original: It is possible, however, to prove the theorem with as much rigor as is looked for in any demonstration involving the theory of limits;
} 
Abaixo segue uma figura mostrando como se calcula o volume da esfera comparando-a com o cone dentro do cilindro. Observe que o cone é formado por duas partes.

Figura 2 - O Princípio aplicado no cálculo do volume de uma esfera

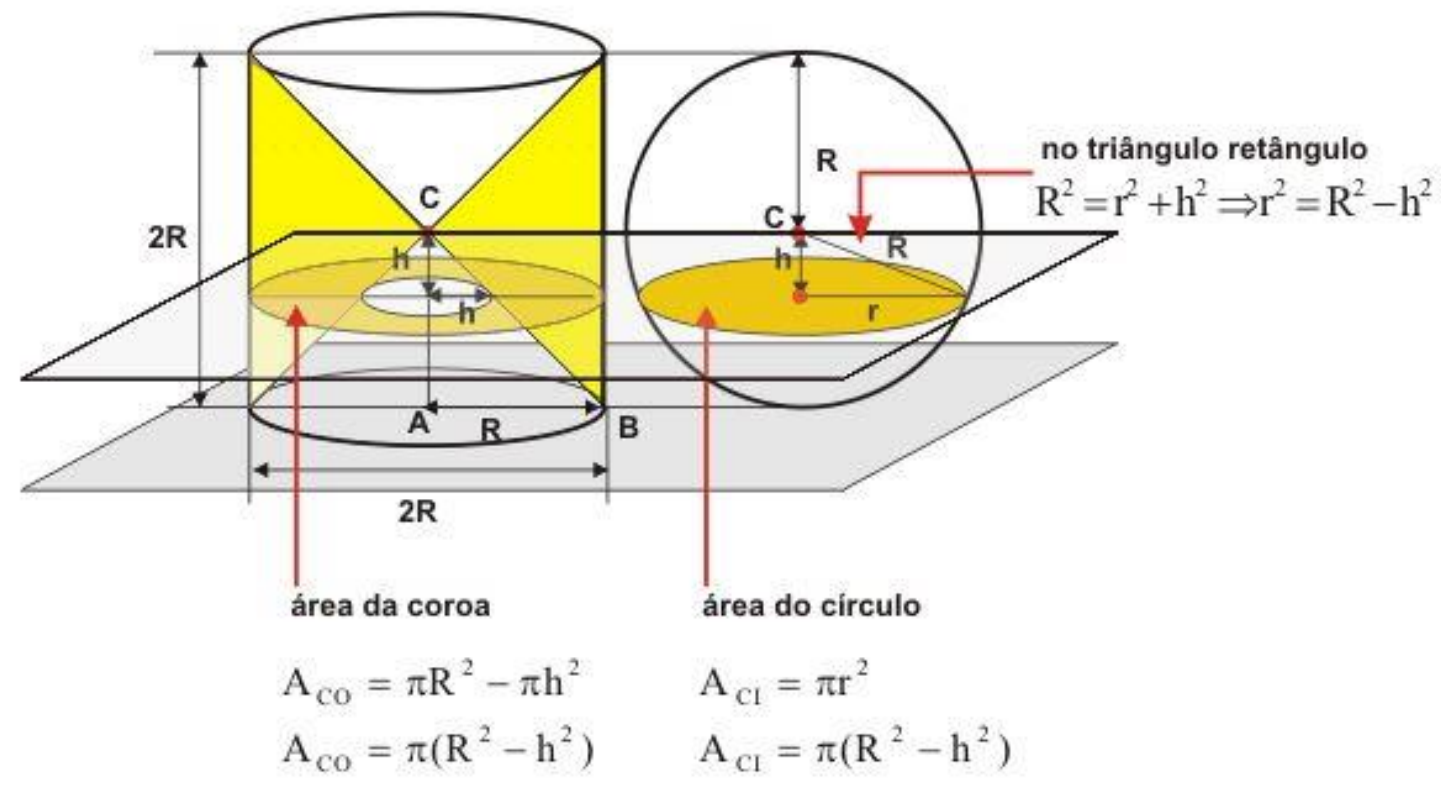

Fonte: <https://www.alfaconnection.pro.br/matematica/geometria/principio-de-cavalieri/calculo-devolumes/>. Acesso em 03 fev. 2021.

Apesar do exemplo acima ser interessante, ele não pertence ao escopo deste trabalho. Nossa pretensão é mostrar como os matemáticos antigos usavam este Princípio para obter grandes resultados. Talvez, um dos mais interessantes seja a descoberta do valor da área exata sob a ciclóide dada por Gilles Personne de Roberval. Com isso, vamos, na próxima seção, fazer uma revisão da construção da ciclóide e, depois, veremos a prova com detalhes dada por Roberval para encontrar a área supracitada.

\section{A ciclóide}

Como este é um texto didático, vamos relembrar nesta seção o que é a ciclóide. Considere, num plano, uma reta $x$ e um círculo de raio qualquer que tangencia esta reta num ponto $O$ fixo neste círculo. Agora, imagine que este círculo descreva um movimento retilíneo e uniforme na mesma direção que a reta, da esquerda para a direita, para fixar 
ideias. Obviamente, à medida que o círculo executa este movimento, o ponto $O$ fixado sobre ele assume várias posições no plano. A ciclóide é a curva formada por todas as posições do ponto $O$ consideradas acima.

Figura 3 - A ciclóide

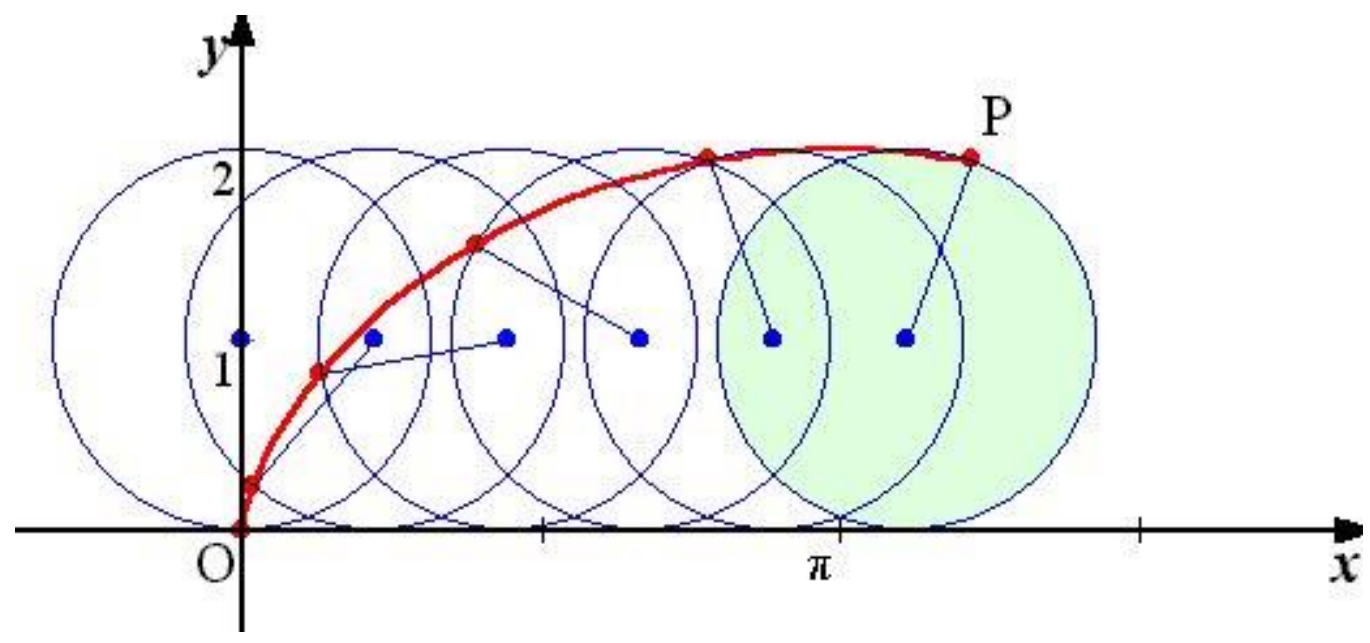

Fonte: <http://www.criced.tsukuba.ac.jp/grapes/pt/image/cycloid01.html>. Acesso: 04 jan. 2021.

Como dissemos no início deste trabalho, um dos objetivos principais é mostrar o Método dos Indivisíveis de Cavalieri, aplicado por Roberval, para calcular a área sob a ciclóide quando o círculo que a descreve "gira" ao longo da reta horizontal. Isto será feito em detalhes na próxima seção.

\section{O Método de Roberval para achar a área sob a ciclóide}

O Método descrito aqui pode ser encontrado, por exemplo, em Fauvel e Gray [1987, p. 376-377]. No entanto, a leitura é complicada e exaustiva. Pretendemos descrevêla de maneira mais elucidativa para o leitor de modo que ele possa visualizar como Roberval era um matemático extremamente habilidoso e apreciar o Método dos Indivisíveis de Cavalieri.

Considere uma reta horizontal e um círculo $A H B$, de diâmetro $A B$ e centro $O$, que se move da esquerda para a direita sempre tangenciando esta reta, isto é, seu centro mantém a mesma distância $r$ do seu raio até a reta. Seja $A$ o ponto de tangência inicial do 
movimento e imagine que o círculo execute meia volta, alcançando o segmento $C D$, com mesma medida de $A B$, e de tal forma que o ponto $A$ atinja a posição ocupada anteriormente pelo ponto $B$. Com isso, teremos claramente que $\operatorname{med}(A C)=\pi r$.

Figura 4 - O movimento do círculo $A H B$

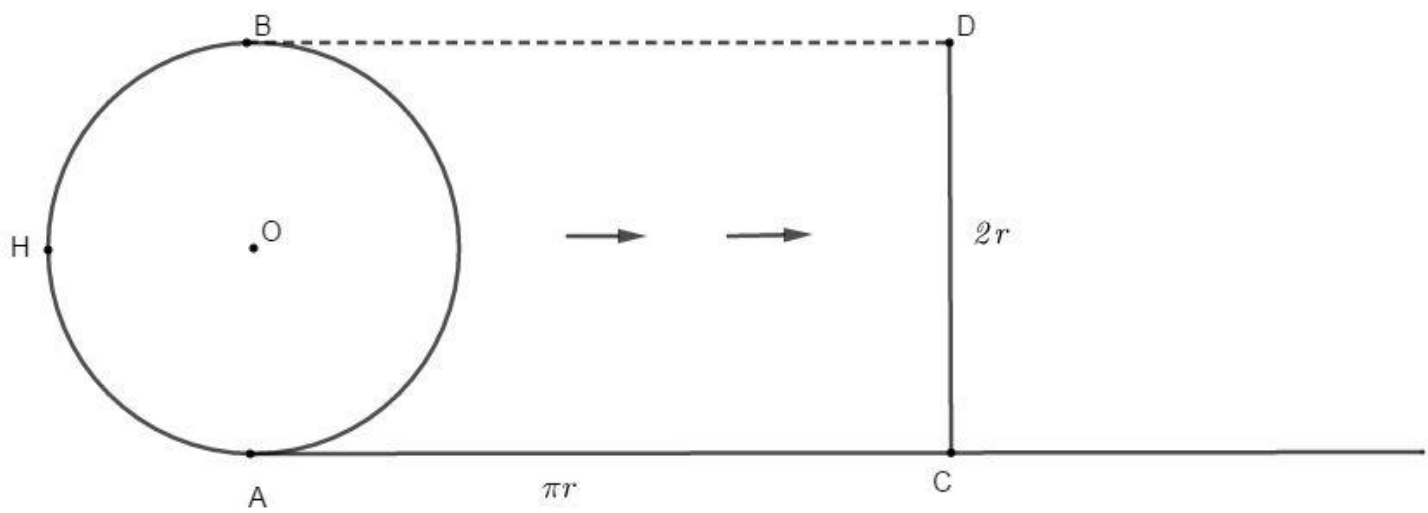

Fonte: Autoral.

Consideraremos, na próxima figura, apenas o semicírculo $A H B$. Para entendê-la, precisamos definir algumas coisas em primeiro lugar.

Considere a divisão do diâmetro $\mathrm{AB}$ em infinitas partes igualmente espaçadas ${ }^{\mathrm{e}} \mathrm{e}$ trace segmentos horizontais de tal forma a interceptarem o semicírculo $A H B$ em seus respectivos pontos.

Para termos uma melhor ideia desse processo, consideremos os pontos 1, 2, 3, 4, 5, 6 e 7 sobre o diâmetro $A B$ e igualmente espaçados. Sejam $E, F, G, H, I, J$ e $K$ os respectivos pontos sobre o semicírculo $A H B$ obtidos pelos segmentos horizontais, como descrito anteriormente.

Agora, vamos tomar os pontos $M, N, O, P, Q, R$ e $S$, sobre o segmento horizontal $A C$, de tal forma que $\operatorname{med}(A M)=\operatorname{med}(\operatorname{arc}(\mathrm{AE})), \operatorname{med}(M N)=\operatorname{med}(\operatorname{arc}(\mathrm{EF})), \ldots$, $\operatorname{med}(S C)=\operatorname{med}(\operatorname{arc}(\mathrm{KB}))$.

\footnotetext{
e É claro que não é possível dividir um segmento em infinitas partes iguais. Deixamos assim no texto para preservar a escrita original e mostrar como os matemáticos da época escreviam. O correto seria dividir o segmento em $n$ partes iguais e tender $n$ para infinito.
} 
Por último, vamos considerar duas curvas descritas pelo ponto $A$, fixado inicialmente, à medida que o movimento do círculo avança.

1. A primeira curva será dada pelo movimento que $A$ percorre ao longo do diâmetro $A B$ da seguinte maneira: quando o ponto $A$ alcançar o ponto $E$ enquanto o círculo "gira", ele se eleva a uma distância igual a $A l$ da reta horizontal inicial; com isso, marcamos o ponto 1, à esquerda do semicírculo, que deve estar sobre um segmento vertical de mesmo tamanho que $A l$ e com a extremidade inferior sobre o ponto $M$. Procedendo de forma análoga e considerando os seguimentos verticais de medidas $A 2, A 3, \ldots, A 7$ com extremidades inferiores sobre os pontos $N, O, \ldots, S$, respectivamente, obtemos a primeira curva desejada.

2. A segunda curva descrita por $A$ será a metade da ciclóide, a curva $A 8 D$.

Figura 5 - As duas curvas percorridas pelo ponto $A$

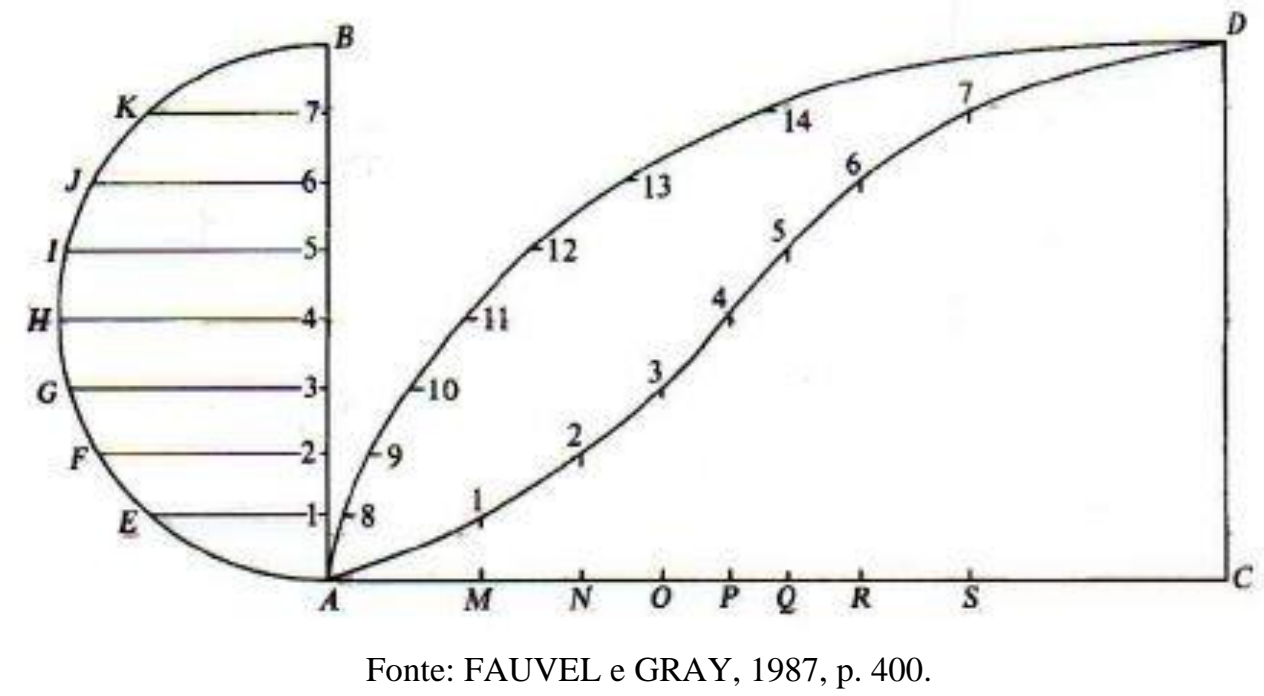

Quando o centro do círculo alcança a posição vertical passando por $M$, pela construção da ciclóide temos que $\operatorname{med}(\operatorname{arc}(A E))=\operatorname{med}(\operatorname{arc}(M 8))$ e, pela construção da primeira curva, $\operatorname{med}(A 1)=\operatorname{med}(M 1)$. Com isso, fica claro que med $(E 1)=$ $\operatorname{med}(81)$. Analogamente, temos que $\operatorname{med}(F 2)=\operatorname{med}(92), \operatorname{med}(G 3)=\operatorname{med}(103) \mathrm{e}$ assim sucessivamente. Isto mostra que os segmentos horizontais traçados no semicírculo possuem a mesma medida dos comprimentos dos segmentos, na sua respectiva reta horizontal, compreendidos na área limitada pela duas curvas contruídas acima. Pelo Pricípio de Cavalieri, segue-se que esta área é igual ao do semicírculo $A H B$, isto é, $\pi r^{2} / 2$. 
Para chegarmos à medida da área sob a ciclóide, basta agora mostarmos que a curva $A 1 D$ divide o paralelogramo $A B C D$ ao meio.

De fato, tomemos, por exemplo, os pontos 1 e 7 sobre o diâmetro $A B$. Eles estão à mesma distância de $A$ e $B$, respectivamente. Logo, temos que $\operatorname{med}(\operatorname{arc}(A E))=$ med $(\operatorname{arc}(K B))$. Por outro lado, sabemos pela construção da figura acima que $\operatorname{med}(\operatorname{arc}(A E))=\operatorname{med}(A M)$ e $\operatorname{med}(\operatorname{arc}(K B))=\operatorname{med}(S C)$. Concluímos, portanto, que $\operatorname{med}(A M)=\operatorname{med}(S C)$.

De forma análoga, vemos que todos os seguimentos horizontais traçados a uma mesma distância de $A C$ e $B D$ a partir do diâmetro $A B$ e do segmento $C D$, respectivamente, interceptam a curva $A 1 D$ de modo a possuírem a mesma medida. Novamente, pelo Princípio de Cavalieri, concluímos que a área do paralelogramo $A B C D$ é dividida ao meio por esta curva, isto é, a área sob a curva $A 1 D$ é igual a $\pi r^{2}$.

Finalmente, juntando este fato à outra área descoberta entre as duas curvas construídas pelo ponto $A$, acabamos por provar que a área sob a metade da ciclóide é $\pi r^{2} / 2+\pi r^{2}=3 \pi r^{2} / 2$ e, consequentemente, a área sob a ciclóide corresponde ao triplo da área do círculo $A H B$ que a gera.

\section{Comentários e conclusões}

Como dissemos no início deste trabalho, o principal objetivo aqui era apresentar algum tema da História da Matemática como um instrumento para o professor da Educação Básica usar em suas aulas para despertar a curiosidade de seus alunos e aumentar seu interesse nesta disciplina.

O tema escolhido foi o Princípio de Cavalieri, pois se trata de um assunto bastante visto no Ensino Básico, mas dado de uma forma "aleatória", sem nenhum compromisso com a aprendizagem significativa de fato por parte dos alunos.

Na questão da escolha dos conteúdos a serem dados em sala de aula, a BNCC (2017) preconiza o seguinte:

[..] decidir sobre formas de organização interdisciplinar dos componentes curriculares e fortalecer a competência pedagógica das equipes escolares para adotar estratégias mais dinâmicas, interativas e colaborativas em relação à gestão do ensino e da aprendizagem. (BRASIL, 2017, p. 16) 
Desta forma, e observando a grande defasagem no currículo dos professores de matemática, procuramos revisar conceitos como a definição da ciclóide, o próprio método de Cavalieri, além de prover um belo resultado de aplicação deste método usando uma linguagem acessível e pretendendo levar o professor-leitor a ir mais além do conteúdo que ele aprendeu enquanto aluno de matemática, apesar das dificuldades impostas pelo sistema educacional deste país.

\section{Referências}

ANDERSEN, K. Cavalieri's Method of Indivisibles. Archive for the History of Exact Sciences, 31, 1985, 291-367.

BRASIL. Base Nacional Comum Curricular. Brasília: MEC, 2017. Disponível em: $<$ http://portal.mec.gov.br/docman/abril-2018-pdf/85121-bncc-ensino-medio/file>. Acesso em: 02 de jan. de 2021.

EVANS, G. W. Cavalieri's Theorem in His Own Words. The America Mathematical Monthly, December 1917, Vol. 10, 447-451.

GRAY J.: The Route to the Calculus - Unit 9. The Open University Course, 1987.

FAUVEL, J.; GRAY J.: The History of Mathematics: A Reader. The Open University, 1987.

LEMOS, C. M.: Os Logaritmos e as suas aplicações nas ciências náuticas - um apontamento histórico. Boletim da SPM 66, maio, Lisboa, 2012, p. 65-104.

MIGUEL, A.: Três estudos sobre história e educação matemática. 1993. [285]f. Tese (doutorado) - Universidade Estadual de Campinas, Faculdade de Educação, Campinas, SP. Disponível em: $<$ http://www.repositorio.unicamp.br/handle/REPOSIP/253114>. Acesso em: 20 jan. 2021. 